\title{
ONDAS ELECTROMAGNÉTICAS EN EL PLANO FRONTERA ENTRE DOS MEDIOS QUIRALES
}

\section{ELECTROMAGNETIC WAVES AT THE PLANE BOUNDARY BETWEEN TWO CHIRAL MEDIA}

\author{
Héctor Torres-Silva ${ }^{1} \quad$ Carlos Villarroel-González ${ }^{2} \quad$ Florentino Jiménez-Muñoz $^{3}$ \\ Recibido 21 de noviembre de 2006, aceptado 6 de marzo de 2007 \\ Received: November 21, 2006 Accepted: March 6, 2007
}

\begin{abstract}
RESUMEN
En este trabajo se estudia la propagación de ondas electromagnéticas en medios quirales isotrópicos y los efectos producidos por un plano frontera entre tales medios. En analogía con el fenómeno de reflexión y refracción de ondas electromagnéticas planas en dieléctricos ordinarios, se estudian los aspectos cinéticos y dinámicos de estos fenómenos, tal como la intensidad de las componentes de onda y el cambio en la polarización de la onda al cruzar la frontera. Como un prerrequisito, mostramos que la solución de onda plana debe ser escrita como una superposición conveniente de las amplitudes quirales circularmente polarizadas en ambos lados de la interfaz. Se presenta el conjunto apropiado de condiciones que la solución debe satisfacer en la frontera y el sistema de ecuaciones que debe ser resuelto para los coeficientes de amplitud de manera de satisfacer las condiciones de frontera. Las ecuaciones pueden ser resueltas explícitamente para algunos casos y configuraciones particulares (por ejemplo, incidencia normal), las características prominentes de estas soluciones pueden ser analizadas en algún detalle. También se presenta un desarrollo de la solución general de las ecuaciones. Este trabajo podría ser aplicado en diseño de microondas en muy altas frecuencias y en líneas de transmisión no simétricas.
\end{abstract}

Palabras clave: Quiral, propagación, ondas, Snell.

ABSTRACT

This work is concerned with the propagation of electromagnetic waves in isotropic chiral media and with the effects produced by a plane boundary between two such media. In analogy with the phenomena of reflection and refraction of plane electromagnetic waves in ordinary dielectrics, the kinematical and dynamical aspects of these phenomena are studied, in situations such as the intensity of the various wave components and the change in the polarization of the wave, as it crosses the boundary. As a prerequisite of this, we show that the plane wave solution must be written as a suitable superposition of the circularly polarized chiral amplitudes on both sides of the interface we elucidate, which is the appropriate set of conditions that the solution must satisfy at the boundary. We set the minimal and complete set of equations that must be solved for the coefficient amplitudes, in order to satisfy the boundary conditions. The equations can be solved explicitly for some particular cases and configurations (e.g., normal incidence), the salient features of those solutions can be analyzed in some detail, and the general solution to the equations is given as well. This work might be applied to the design of very high frequency microwaves and non symmetrical transmission lines.

Keywords: Chiral, propagation, waves, Snell.

\section{INTRODUCCIÓN}

Los problemas relacionados con la propagación de las ondas electromagnéticas en un medio homogéneo, incluyendo los de reflexión y refracción en la interfaz entre dos medios, forman un conjunto tradicional en la electrodinámica clásica y se discuten en cualquier libro de texto sobre el tema [1]. Uno menos familiar es el problema de la propagación de una onda electromagnética en un medio quiral. En tal medio, la polarización de la onda permite que las dos ondas polarizadas circularmente se propaguen con velocidades diferentes. Un medio quiral,

1 Instituto de Alta Investigación, Universidad de Tarapacá, Casilla 6-D. Arica, Chile.

2 Escuela Universitaria de Ingeniería Eléctrica - Electrónica, Universidad de Tarapacá. Casilla 6-D. Arica, Chile. cvillar@uta.cl

3 E.U.I.T. de Telecomunicación, Universidad Politécnica de Madrid. Campus Sur UPM, Ctra. de Valencia, km. 7-28031. Madrid, España. 
también llamado "ópticamente activo", puede existir en variadas situaciones. Por ejemplo, un medio material puede transformarse en ópticamente activo bajo la influencia de un campo externo, tal como un campo magnético (el bien conocido efecto Faraday discutido en muchos libros de texto de física de plasma [2]) o por un control de onda electromagnética plana [3]. Otro tipo de medio exhibe el fenómeno quiral naturalmente [4]. Recientemente, materiales compuestos artificialmente, tipo metamateriales, han sido producidos con propiedades similares [5].

Varias aproximaciones han sido propuestas en la literatura para estudiar las propiedades electromagnéticas de tales medios ópticamente activos [4,6-14].

El formalismo desarrollado en [15] se refiere sólo a un medio plasmático homogéneo. Los problemas de fluido plasmático, asociados con lo electromagnético, tratan este tipo de problema. En particular, aquí estudiaremos en detalle el problema de la reflexión y refracción en la interfaz, para el caso en que uno o ambos medios semiinfinitos en cualquier lado de la frontera exhiban la propiedad de quiralidad.

El trabajo está organizado como sigue. En la sección "Parametrización de las Fuentes Inducidas" se resume la parametrización de la carga inducida y la corriente para un medio quiral homogéneo. Entonces se extiende para tratar un medio no homogéneo y se considera en particular el sistema que consiste de dos medios homogéneos semiinfinitos separados por un plano frontera, que es lo que interesa en el presente trabajo. Para establecer la etapa, para el estudio de la propagación de la onda que cruza la frontera, entre dos medios quirales, en la siguiente sección revisamos brevemente las características principales de la propagación de una onda plana en un medio homogéneo quiral, su relación de dispersión. En la sección siguiente establecemos el problema de la reflexión y refracción en el plano interfaz entre dos medios quirales, en términos de un conjunto de ecuaciones que deben ser resueltas, para los varios coeficientes de amplitudes que aparecen en la solución de onda plana descompuesta de las ecuaciones de Maxwell. La última sección contiene algunas conclusiones y observaciones.

\section{PARAMETRIZACIÓN DE LAS FUENTES INDUCIDAS}

Este trabajo trata exclusivamente con materiales que son isotrópicos, se asume que los campos son tales que el material responde linealmente, se utiliza el sistema de unidades denominado Heaviside-Lorentz.

\section{Medio no dispersivo}

Por orientación, consideremos primero un medio no dispersivo. Escribiendo la carga inducida y la densidades de corriente en la forma usual

$$
\begin{aligned}
& \rho_{\text {ind }}=-\vec{\nabla} \cdot \vec{P}, \\
& \vec{j}_{\text {ind }}=\frac{\partial \vec{P}}{\partial t}+\vec{\nabla} \times \vec{M},
\end{aligned}
$$

La relación entre la polarización eléctrica y magnética, $\vec{P}$ y $\vec{M}$, en el caso quiral es más general que la usual. Dicha relación es [9]:

$$
\begin{aligned}
& \vec{P}=(\varepsilon-1) \vec{E}+\zeta \vec{B} \\
& \vec{M}=\left(1-\mu^{-1}\right) \vec{B}-\zeta \vec{E},
\end{aligned}
$$

En particular, la isotropía del medio implica que las cantidades $\varepsilon, \mu, \zeta$ son escalares más que tensores, y la naturaleza no dispersiva del medio implica que esas mismas cantidades son constantes independientes de las coordenadas. El par homogéneo de las ecuaciones de Maxwell, es:

$$
\begin{gathered}
\vec{\nabla} \cdot \vec{B}=0 \\
\vec{\nabla} \times \vec{E}=-\frac{\partial \vec{B}}{\partial t}
\end{gathered}
$$

y en lugar de la ec. (1) se tiene

$$
\begin{gathered}
\rho_{\text {ind }}=-(\varepsilon-1) \vec{\nabla} \cdot \vec{E} \\
\vec{j}_{\text {ind }}=(\varepsilon-1) \frac{\partial \vec{E}}{\partial t}+\left(1-\frac{1}{\mu}\right) \vec{\nabla} \times \vec{B}-\zeta \vec{\nabla} \times \vec{E} .
\end{gathered}
$$

\section{Medio homogéneo no dispersivo}

Mientras las ecuaciones anteriores se refieren a un medio no dispersivo, consideraciones similares y las mismas conclusiones se tienen para un medio dispersivo, pero también homogéneo. En un medio dispersivo, la relación entre $\vec{P}, \vec{M}$ y los campos electromagnéticos no es local. Por lo tanto, expresiones como $\varepsilon \vec{E}$ se interpretarían en la forma

$$
\varepsilon \vec{E} \rightarrow \hat{\varepsilon} \vec{E} \equiv \int d^{4} x^{\prime} \varepsilon\left(x, x^{\prime}\right) \vec{E}\left(x^{\prime}\right)
$$

Se procederá en forma similar con los otros términos envueltos $\mu$ y $\zeta$ (se usará la notación compacta tetradimensional $x=(t, \vec{x})$ en los argumentos de las funciones). 
Sin embargo, la suposición de que el medio es homogéneo implica que las funciones $\varepsilon, \mu$ y $\zeta$ dependen solamente de la distancia $\vec{x}-\vec{x}^{\prime}$ y, además, el principio de causalidad requiere que la dependencia del tiempo esté en la variable t-t' y no en t y t' separadamente. En este caso las relaciones tales como las de la ec. (7) se convierten en simples relaciones algebraicas en el espacio de Fourier. El argumento y la parametrización utilizados anteriormente se mantienen, si cada símbolo se toma para ser la transformada de Fourier de la correspondiente coordenada espacial variable, y si se hace la identificación correspondiente en el espacio de Fourier

$$
\begin{aligned}
& \vec{\nabla} \rightarrow i \vec{k}, \\
& \frac{\partial}{\partial t} \rightarrow-i \omega .
\end{aligned}
$$

Las fuentes inducidas son parametrizadas como

$$
\begin{aligned}
& \rho_{\text {ind }}(\omega, \vec{k})=-i(\varepsilon-1) \vec{k} \cdot \vec{E}, \\
& \vec{j}_{\text {ind }}(\omega, \vec{k})=-i(\varepsilon-1) \omega \vec{E}+i\left(1-\mu^{-1}\right) \vec{k} \times \vec{B}-i \zeta \vec{k} \times \vec{E},
\end{aligned}
$$

y las correspondientes expresiones en coordenadas espaciales son

$$
\begin{gathered}
\rho_{\text {ind }}=-\vec{\nabla} \cdot[(\hat{\varepsilon}-1) \vec{E}], \\
\vec{j}_{\text {ind }}=\frac{\partial}{\partial t}[(\hat{\varepsilon}-1) \vec{E}]+\vec{\nabla} \times\left[\left(1-\frac{1}{\hat{\mu}}\right) \vec{B}\right]-\vec{\nabla} \times(\zeta \vec{E})
\end{gathered}
$$

El último término en la ec. (10) puede ser escrito en términos de $\vec{B}$ usando la siguiente relación

$$
\vec{\nabla} \times(\zeta \vec{E})=-\frac{\partial(\zeta \vec{B})}{\partial t},
$$

lo que es una consecuencia de la ley de Faraday, como puede ser visto muy simplemente en el espacio de Fourier. En el espacio de coordenadas se probó que una vez advertido eso, para un medio homogéneo como el que se ha considerado, podemos utilizar manipulaciones tales como

$$
\begin{gathered}
\frac{\partial}{\partial x_{i}} \int d^{4} x \zeta\left(x-x^{\prime}\right) \vec{E}\left(x^{\prime}\right)=-\int d^{4} x\left(\frac{\partial}{\partial x_{i}^{\prime}} \zeta\left(x-x^{\prime}\right)\right) \vec{E}\left(x^{\prime}\right) \\
=\int d^{4} x \zeta\left(x-x^{\prime}\right) \frac{\partial}{\partial x_{i}^{\prime}} \vec{E}\left(x^{\prime}\right)
\end{gathered}
$$

donde se ha integrado por partes.
Así, en ausencia de fuentes externas, las ecuaciones de Maxwell en un medio quiral homogéneo consisten del par dado en las ec. (3) y (4), aumentado por

$$
\vec{\nabla} \cdot(\vec{D})=0,
$$

con $\vec{D}=\varepsilon \vec{E}+\zeta \vec{B}$

$$
\vec{\nabla} \times\left(\frac{1}{\hat{\mu}} \vec{B}+\zeta \vec{E}\right)-\frac{\partial}{\partial t}(\hat{\varepsilon} \vec{E})=0,
$$

donde $\zeta$ es la admitancia quiral.

Si las fuentes externas no son cero, éstas podrían aparecer en el lado derecho de estas dos últimas ecuaciones [10].

\section{Dos medios quirales homogéneos con una interfaz}

El tipo de sistema que concierne a este trabajo es, a saber, dos medios homogéneos semiinfinitos, separados por un plano interfaz.

Definiendo el plano frontera por $\mathrm{z}=0$. En el límite idealizado que lleva al plano frontera a ser infinitesimalmente delgado, entonces podemos representar la función de separación $\hat{\eta}$ en la forma

$$
\hat{\eta}=\eta \theta(-z)+\eta^{\prime} \theta(z)
$$

Sin embargo, en cualquier lado de la frontera (esto es, para $z \neq 0$ ), la función es cero dado que se está considerando ambos medios semiinfinitos como homogéneos. Así, $\hat{\eta}=0$ en todas partes.

La excepción a este argumento surge si la situación es tal que el plano frontera no puede ser tomado como infinitamente delgado, en su lugar se debe tener en cuenta el hecho que tiene algún espesor $\delta$ distinto de cero. Ciertamente, en la transición desde un material al otro, el medio es no homogéneo y en esa región $\hat{\eta}$ puede no ser cero. Si miramos los efectos físicos que ocurren en cualquier medio en distancias, digamos $|z| \sim L$ con $L \gg \delta$, entonces los efectos debidos al valor distinto de cero de $\hat{\eta}$ en la región de transición son del orden de $\delta / L$ y son efectos de superficie. En puntos que están cerca al plano frontera $(|z| \sim \delta)$ tales efectos pueden ser observables, pero los efectos se vuelven insignificantes cuando $\delta / L \rightarrow 0$, como sería entonces en ese caso, donde la región de transición puede ser idealizada para ser infinitamente delgada y entonces la representación dada en la ec. (15) es válida. 


\section{PROPAGACIÓN DE UNA ONDA EN UN MEDIO HOMOGÉNEO ÓPTICAMENTE ACTIVO}

\section{Relación de dispersión}

Para propósitos ilustrativos, se considera una onda electromagnética en estado estacionario (monocromática) propagándose a lo largo de una dirección dada, la que se considerará como el eje z. Así, el vector potencial es de la forma

$$
\vec{A}(z, t)=e^{-i \omega t} \vec{a}(z),
$$

donde $\vec{a}(z)$ debe ser elegido tal que el vector potencial $\vec{A}(z, t)$ satisfaga las ecuaciones de Maxwell con las apropiadas condiciones de frontera. Considerando este hecho en las ecs. (13) y (14), sigue que la ecuación de onda es satisfecha solamente por las ondas polarizadas circularmente cuyos vectores de polarización son definidos por

$$
\hat{e}_{ \pm} \equiv \frac{1}{\sqrt{2}}\left(\hat{u}_{x} \pm i u_{y}\right)
$$

donde de denota por $\hat{u}_{x}, \hat{u}_{y}$ y $\hat{u}_{z}$ a los vectores unitarios a lo largo de las direcciones $\mathrm{x}, \mathrm{y} \mathrm{y} \mathrm{z}$, respectivamente. Además, estos estados de polarización satisfacen las relaciones de dispersión

$$
n_{\sigma}(\zeta)\left(\omega, K_{\sigma}\right)= \pm \zeta \mu+\sqrt{\zeta^{2} \mu^{2}+\varepsilon \mu}
$$

donde las funciones índice refractivo $n_{ \pm}$que aparecen en la ec. (18) están dadas en términos de la función dieléctrica $(\varepsilon)$, la permeabilidad magnética $(\mu)$ y la constante de actividad $(\zeta)$ del medio por

$$
n_{ \pm}^{2}(\zeta)-2 \zeta \mu n_{ \pm}(\zeta)=\varepsilon \mu
$$

Mientras el índice de refracción puede en general ser complejo, aun sin el término $\zeta$. En este trabajo se considera solamente sistemas para los cuales el índice es real, es decir, el medio no es absorbente. Esto implica, en particular, que $\zeta$ es puramente imaginario. Esto, en cambio, tiene implicaciones en las propiedades microscópicas del sistema con respecto a las simetrías espacio-tiempo discretas, que fueron consideradas ampliamente en [15].

Para tener una relación lineal para el índice de refracción se realizan las siguientes transformaciones:

$$
\begin{aligned}
& i \omega \zeta=2 \omega^{2} \varepsilon T /\left(1-k_{0}^{2} T^{2}\right), \\
& \varepsilon=\varepsilon^{*} /\left(1-k_{0}^{2} T^{2}\right), \\
& \mu=\mu^{*}
\end{aligned}
$$

Entonces se tiene

$$
n_{ \pm}= \pm n_{0} /\left(1 \mp k_{0} T^{*}\right)=\frac{K_{\sigma}(\omega)}{\omega} .
$$

Este es el resultado fundamental de esta sección y que permite obtener resultados en forma más directa.

\section{Onda polarizada elípticamente}

La generalización obvia de las formas especiales anteriores es la superposición

$$
\vec{A}(z, t)=e^{-i \omega t}\left[A_{+} e^{i z K_{+}} \hat{e}_{+}+A_{-} e^{i z K_{-}} \hat{e}_{-}\right],
$$

la cual contiene una mezcla desigual de ambas polarizaciones circulares. Una simple representación geométrica de esta solución está dada por la siguiente construcción. En el punto $\mathrm{z}=0$, la dirección del campo eléctrico está dada por el vector

$$
\vec{\varepsilon}(z=0, t)=\left(A_{+}+A_{-}\right) \hat{u}_{x} \cos \omega t+\left(A_{+}-A_{-}\right) \hat{u}_{y} \operatorname{sen} \omega t
$$

Esto es, el vector de polarización rota en una elipse, con el eje de la elipse ubicado a lo largo de las direcciones $\mathrm{x}$ e $\mathrm{y}$. Para un punto arbitrario $\mathrm{z} \neq 0$, escribimos la ec. (19) en la forma equivalente

$$
\vec{A}(z, t)=e^{-i \omega t} e^{i \Delta(z)}\left[A_{+} e^{-i \theta(z)} \hat{e}_{+}+A_{-} e^{i \theta(z)} \hat{e}_{-}\right]
$$

donde $\theta(z)$ y y $\Delta(z)$ están definidas anteriormente. La dirección del campo eléctrico se obtiene fácilmente de aquí para ser representada por el vector

$$
\vec{\varepsilon}(z, t)=\left(A_{+}+A_{-}\right) \cos (\omega t-\Delta(z)) \hat{u}_{x^{\prime}}+\left(A_{+}-A_{-}\right) \operatorname{sen}(\omega t-\Delta(z)) \hat{u}_{y^{\prime}}
$$

donde se ha definido

$$
\begin{aligned}
& u_{x^{\prime}}=\cos \theta(z) \hat{u}_{x}+\operatorname{sen} \theta(z) \hat{u}_{y} \\
& u_{y^{\prime}}=-\operatorname{sen} \theta(z) \hat{u}_{x}+\cos \theta(z) \hat{u}_{y} .
\end{aligned}
$$

Por lo tanto, en un punto $\mathrm{z}=\mathrm{L}$ la polarización es también elíptica, pero en contraste con el fenómeno de polarización elíptica estándar en un medio ordinario, la elipse misma ha rotado un ángulo $\theta(L)$. Además, el ángulo de polarización adquiere una fase negativa $\Delta(L)$ relativa al eje x'. 
En las siguientes secciones se extenderán estos ejemplos al caso en el cual la onda cruza el plano interfaz entre dos medios, cada uno de los cuales es individualmente descrito tal como se ha explicado anteriormente. El tratamiento de este problema debe necesariamente tomar en cuenta, entre otros temas, los aspectos cinéticos y dinámicos de la reflexión y refracción de la onda en el plano frontera.

\section{Reflexión y refracción de una onda polarizada circularmente}

Se considerará una onda electromagnética incidente sobre un plano frontera como se muestra esquemáticamente en la figura 1 , en ella se definen las regiones 1 y 2 . La homogeneidad del espacio en las direcciones xy permite buscar las soluciones (onda plana) en la forma

$$
e^{-i \omega t} e^{i \vec{k}_{\perp} \cdot \vec{x}} \vec{A}(z)
$$

Para un medio ordinario (no quiral), un planteamiento conveniente es

$$
\vec{A}(z)=\left[\vec{a} e^{i k_{\|}^{z}}+\vec{b} e^{-i k_{\|}^{z}}\right] \Theta(-z)+\left[\vec{c} e^{i k_{\|}^{z}}\right] \Theta(z)
$$

La forma elegida en la ec. (25) corresponde a la situación física en la cual la onda es incidente desde la izquierda (con ninguna reflexión desde el lado derecho distante, como sería en un medio semiinfinito), tal como se representa en la figura 1.

Aquí se encuentra la primera divergencia del tratamiento estándar cuando se considera un medio quiral. A saber, mientras se pueda hacer que la componente incidente consista solamente de una de las dos polarizaciones, en general la reflejada y la refractada consistirán de una superposición de los dos modos propagados, los cuales en el presente caso tienen diferentes longitudes de onda. Por ejemplo, si se descompone el vector de la onda refractada en sus componentes perpendicular y paralela (relativo al plano z $=0) \vec{k}^{\prime}=\left(\vec{k}_{\perp}, k_{\|}^{\prime}\right)$, entonces para valores fijos de $\omega$ y $\vec{k}_{\perp}$ existe de hecho un valor diferente de la componente paralela para cada polarización, dada por $\sqrt{K_{\tau}^{\prime 2}-k_{\perp}^{2}}$, de manera que las dos componentes de polarización viajan en direcciones diferentes.

Con esto en mente, se escribirá la solución propuesta correspondiente a una onda con polarización definida $\sigma= \pm$ incidente desde la izquierda, en la forma de la ec. (26).
(I)

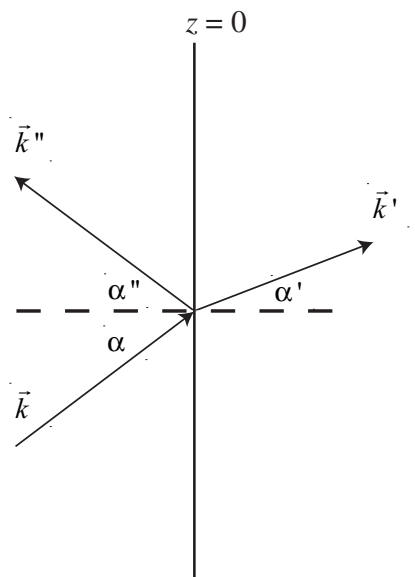

(II)

Figura 1. Onda electromagnética incidente sobre un plano frontera.

$$
\vec{A}(\vec{x}, t)=e^{-i \omega t}\left[\vec{A}_{I} \Theta(-z)+\vec{A}_{I I} \Theta(z)\right],
$$

donde $\vec{A}_{I}$, que contiene las componentes incidente y reflejada, se toma como

$$
\vec{A}_{I}=a_{\sigma} \hat{e}_{\sigma} e^{i \vec{k}_{\sigma} \cdot \vec{x}}+\sum_{\tau= \pm} a_{\tau}^{\prime \prime \hat{e}_{\tau}^{\prime \prime}} e^{i \vec{k}_{\tau}^{\prime \prime} \cdot \vec{x}},
$$

mientras la componente refractada $\vec{A}_{I I}$ es

$$
\vec{A}_{I I}=\sum_{\tau= \pm} a_{\tau}^{\prime} \hat{e}_{\tau}^{\prime} e^{i \vec{k}_{\tau}^{\prime} \cdot \vec{x}}
$$

Los vectores de onda en las expresiones para esas ondas pueden ser escritos como

$$
\begin{aligned}
& \vec{k}_{\sigma}=\left(\vec{k}_{\perp}, k_{\sigma \|}\right), \\
& \vec{k}_{\tau}^{\prime}=\left(\vec{k}_{\perp}, k_{\tau \|}^{\prime}\right), \\
& \vec{k}_{\tau}^{\prime \prime}=\left(\vec{k}_{\perp},-k_{\tau \|}\right)
\end{aligned}
$$

compartiendo un valor común de la componente perpendicular. Para el mismo valor fijo de $\omega$, denotamos por $K_{\sigma}(\omega)$ y $K_{\sigma}^{\prime}(\omega)$ las soluciones de las relaciones de dispersión en las dos regiones caracterizadas por funciones de índice refractivo $n_{ \pm} \mathrm{y} n_{ \pm}^{\prime}$. Haciendo referencia a los ángulos mostrados en la figura 1, las componentes paralelas para los diferentes vectores de onda pueden ser escritas como

$$
\begin{aligned}
& k_{\sigma\|\|}=K_{\sigma} \cos \alpha \\
& k_{\tau \|}^{\prime}=K_{\tau}^{\prime} \cos \alpha_{\tau}^{\prime}, \\
& k_{\tau \|}^{\prime \prime}=K_{\tau} \cos \alpha_{\tau}^{\prime \prime},
\end{aligned}
$$


donde

$$
\begin{aligned}
& k_{\tau \|}^{\prime}=\sqrt{K_{\tau}^{\prime 2}-k_{\perp}^{2}} \\
& k_{\tau \|}^{\prime \prime}=\sqrt{K_{\tau}^{2}-k_{\perp}^{2}} .
\end{aligned}
$$

De manera de especificar los vectores de polarización unívocamente, definimos $\hat{e}_{1}$ para que este en la dirección de $\vec{k}_{\perp} \times \hat{u}_{z}$. Haciendo referencia a la figura 1 , apunta perpendicular a la página, alejándose del observador. Tomamos esto para que también sea la dirección del eje $\mathrm{x}, \mathrm{y}$ definimos el eje $\mathrm{y}$ por

$$
\hat{u}_{y}=\hat{u}_{z} \times \hat{u}_{x}
$$

el cual es la dirección vertical en la representación de la figura 1. Entonces, para los vectores de polarización, tenemos

$$
\hat{e}_{1}^{\prime}=\hat{e}_{1}^{\prime \prime}=\hat{e}_{1}=\hat{u}_{x},
$$

mientras

$$
\begin{aligned}
& \hat{e}_{2}=\hat{k} \times \hat{e}_{1}, \\
& \hat{e}_{2 \tau}^{\prime}=\hat{k}_{\tau}^{\prime} \times \hat{e}_{1}, \\
& \hat{e}_{2 \tau}^{\prime \prime}=\hat{k}_{\tau}^{\prime \prime} \times \hat{e}_{1}
\end{aligned}
$$

donde $\hat{k}, \hat{k}_{\tau}^{\prime}$ y $\hat{k}_{\tau}^{\prime \prime}$ son los vectores unitarios a lo largo de $\vec{k}_{\sigma}, \vec{k}_{\tau}^{\prime}$ y $\vec{k}_{\tau}^{\prime \prime}$, respectivamente. Nótese que, mientras la dirección de la onda incidente es fija (fijada en este análisis), las componentes reflejada y refractada consisten cada una de dos ondas polarizadas que viajan en direcciones diferentes, como ya se ha observado. Con la elección de ejes especificada anteriormente se tiene,

$$
\begin{aligned}
& \hat{k}=\cos \alpha \hat{u}_{z}+\operatorname{sen} \alpha \hat{u}_{y}, \\
& \hat{k}_{\tau}^{\prime}=\cos \alpha_{\tau}^{\prime} \hat{u}_{z}+\operatorname{sen} \alpha_{\tau}^{\prime} \hat{u}_{y}, \\
& \hat{k}_{\tau}^{\prime \prime}=-\cos \alpha_{\tau}^{\prime \prime} \hat{u}_{z}+\operatorname{sen} \alpha_{\tau}^{\prime \prime} \hat{u}_{y},
\end{aligned}
$$

y usando la ec. (34),

$$
\begin{aligned}
& \hat{e}_{2}=-\operatorname{sen} \alpha \hat{u}_{z}+\cos \alpha \hat{u}_{y} \\
& \hat{e}_{2 \tau}^{\prime}=-\operatorname{sen} \alpha_{\tau}^{\prime} \hat{u}_{z}+\cos \alpha_{\tau}^{\prime} \hat{u}_{y} \\
& \hat{e}_{2 \tau}^{\prime \prime}=-\operatorname{sen} \alpha_{\tau}^{\prime \prime} \hat{u}_{z}-\cos \alpha_{\tau}^{\prime \prime} \hat{u}_{y} .
\end{aligned}
$$

Los vectores de polarización circular que intervienen en la ec. (30) están definidos por

$$
\begin{aligned}
& \hat{e}_{\sigma}=\frac{1}{\sqrt{2}}\left(\hat{e}_{1}+i \sigma \hat{e}_{2}\right), \\
& \hat{e}_{\tau}^{\prime}=\frac{1}{\sqrt{2}}\left(\hat{e}_{1}+i \tau \hat{e}_{2 \tau}^{\prime}\right),
\end{aligned}
$$

$$
\hat{e}_{\tau}^{\prime \prime}=\frac{1}{\sqrt{2}}\left(\hat{e}_{1}+i \tau \hat{e}_{2 \tau}^{\prime \prime}\right)
$$

y satisfacen

$$
\begin{aligned}
& \hat{e}_{\sigma} \cdot \vec{k}_{\sigma}=0, \\
& \hat{e}_{\tau}^{\prime \prime} \cdot \vec{k}_{\tau}^{\prime \prime}=0, \\
& \hat{e}_{\tau}^{\prime} \cdot \vec{k}_{\tau}^{\prime}=0 .
\end{aligned}
$$

En esta forma se asegura que las funciones

$$
e^{-i \omega t} \vec{A}_{X} \quad(X=I, I I)
$$

satisfacen las ecuaciones de Maxwell en cada región.

El hecho de que los vectores de onda tengan la misma componente transversal, como se indica en la ec. (33), implica la relación familiar (ley de Snell) entre los ángulos de incidencia y refracción,

$$
\frac{\operatorname{sen} \alpha_{\tau}^{\prime}}{\operatorname{sen} \alpha}=\frac{K_{\sigma}}{K_{\tau}^{\prime}}=\frac{n_{\sigma}}{n_{\tau}^{\prime}}
$$

y la relación análoga para la onda reflejada

$$
\begin{aligned}
& \alpha_{\tau}^{\prime \prime}=\alpha \quad(\tau=\sigma) \\
& \frac{\operatorname{sen} \alpha_{\tau}^{\prime \prime}}{\operatorname{sen} \alpha}=\frac{K_{\sigma}}{K_{\tau}}=\frac{n_{\sigma}}{n_{\tau}} \quad(\tau \neq \sigma) .
\end{aligned}
$$

Así, para un valor dado de $\omega$ y $\alpha$, resulta que el vector de onda $\vec{k}_{\sigma}$, de la componente incidente, está completamente especificado. Los vectores de onda $\vec{k}_{\tau}^{\prime \prime}$ y $\vec{k}_{\tau}^{\prime}$, de las componentes reflejada y refractada, también están completamente determinados, al igual que los vectores de polarización. Las únicas cantidades por ser aún determinadas son las amplitudes $a_{\tau}^{\prime}, a_{\tau}^{\prime \prime}$ en la ec. (30).

De las ecs. (39) y (40) los efectos familiares tales como la reflexión interna total pueden ser deducidos, en la forma en la que ellos aplican a la presente situación. Así, por ejemplo, si las constantes dieléctricas son tales que $K_{\sigma}>K_{\tau}^{\prime}$, existe un ángulo incidente máximo $\alpha_{\tau \max }$, determinado por

$$
\left(\operatorname{sen} \alpha_{\tau}\right)_{\max }=\frac{K_{\tau}^{\prime}}{K_{\sigma}},
$$

por sobre el cual la onda refractada con polarización $\tau$ se propaga paralela a la superficie $\left(k_{\tau \|}^{\prime}=0\right)$, pero no penetra la región II. 
En general, el cuadro que emerge es el siguiente. La onda incidente, la cual tiene una polarización circular definida, se separa en la frontera en una onda reflejada y otra refractada, cada una de las cuales es una superposición de las dos polarizaciones circulares. Sin embargo, los dos modos polarizados circularmente no viajan en la misma dirección como una consecuencia del hecho de que sus vectores de onda tienen la misma componente transversal, pero una componente longitudinal diferente. La determinación de la mezcla relativa de los dos modos de polarización en la onda reflejada y en la refractada es precisamente uno de los aspectos dinámicos que discutiremos más adelante.

\section{SOLUCIÓN DE ECUACIÓN DE ONDA EN LA FRONTERA}

Algunos de los temas dinámicos que deseamos considerar envuelven: (i) encontrar las amplitudes de las componentes reflejada y refractada de la onda para una amplitud incidente dada, (ii) determinar cómo la polarización de la onda cambia cuando cruza la frontera. En contraste con las propiedades cinéticas que consideramos en la sección previa, estos aspectos dinámicos dependen de la naturaleza específica de las condiciones de frontera en la interfaz. Estos últimos son determinados por el requerimiento de que la solución dada en la ec. (30) satisface las ecuaciones de Maxwell en la interfaz.

\section{Condiciones de frontera}

Tal como ya se ha mencionado, las relaciones de dispersión junto con las condiciones dadas en la ec. (36) implica que la función $\vec{A}(\vec{x}, t)$ definida en la ec. (34) satisface las ecuaciones de Maxwell en cada región separadamente. Sin embargo, aún tenemos que asegurar que las ecuaciones se satisfacen en la frontera entre las dos regiones. Este requerimiento produce aún más condiciones. ¿Son las mismas que para las ecuaciones ordinarias? o, ¿El término constante de actividad $\zeta$ las modifica de alguna manera? Esto se responde calculando el campo eléctrico y magnético asociado con el vector potencial dado en la ec. (25). Las fórmulas estándares son

$$
\begin{aligned}
& \vec{E}=-\frac{\partial \vec{A}}{\partial t}, \\
& \vec{B}=\vec{\nabla} \times \vec{A},
\end{aligned}
$$

en términos del potencial magnético se tiene

$$
\vec{E}=e^{-i \omega t}\left[\vec{E}_{I} \Theta(-z)+\vec{E}_{I I} \Theta(z)\right]
$$

$$
\vec{B}=e^{-i \omega t}\left[\vec{B}_{I} \Theta(-z)+\vec{B}_{I I} \Theta(z)+\delta(z) \hat{u}_{z} \times\left(\vec{A}_{I I}-\vec{A}_{I}\right)\right]
$$

donde

$$
\begin{aligned}
& \vec{E}_{X} \equiv i \omega A_{X}, \\
& \vec{B}_{X} \equiv \vec{\nabla} \times \vec{A}_{X} \quad(X=I, I I) .
\end{aligned}
$$

Dado que los campos deben ser finitos en todas partes (de otro modo las ecuaciones de Maxwell podrían no ser satisfechas), el último término en la ec. (40) debe ser cero, lo cual produce la siguiente condición en los vectores de polarización

$$
\hat{u}_{z} \times\left[a_{\sigma} \hat{e}_{\sigma}+\sum_{\tau}\left(a_{\tau}^{\prime \prime} \hat{e}_{\tau}^{\prime \prime}-a_{\tau}^{\prime} \hat{e}_{\tau}^{\prime}\right)\right]=0
$$

Las relaciones que quedan se derivan de las ecuaciones de Maxwell, las cuales fueron presentadas en las ecs. (3), (4), (13) y (14) en la sección "Parametrización de las fuentes inducidas". El argumento para obtener las condiciones implícitas es similar al usado para llegar a la ec. (43). Por ejemplo, considerar la ecuación para el campo eléctrico.

$$
\hat{\varepsilon} \vec{E}=e^{-i \omega t}\left[\varepsilon \vec{E}_{I} \Theta(-z)+\varepsilon^{\prime} \vec{E}_{I I} \Theta(z)\right],
$$

entonces se expresa

$$
\begin{aligned}
\vec{\nabla} \cdot(\hat{\varepsilon} \vec{E}) & =e^{-i \omega t}\left[\Theta(-z) \vec{\nabla} \cdot\left(\varepsilon \vec{E}_{I}\right)+\Theta(z) \vec{\nabla} \cdot\left(\varepsilon^{\prime} \vec{E}_{I I}\right)+\right. \\
& \left.+\delta(z) \hat{u}_{z} \cdot\left(\varepsilon^{\prime} \vec{E}_{I I}-\varepsilon \vec{E}_{I}\right)\right]
\end{aligned}
$$

Los primeros dos términos en el lado derecho son idénticos a cero dado que no existe carga libre en ninguna región. Así, la condición de que la divergencia de $\hat{\varepsilon} \vec{E}$ sea cero, en todas partes, requiere que el coeficiente de $\delta(z)$ en la ec. (48) sea cero. Esto produce la relación

$$
\hat{u}_{z} \cdot\left[\varepsilon a_{\sigma} \hat{e}_{\sigma}+\sum_{\tau}\left(\varepsilon a_{\tau}^{\prime \prime} \hat{e}_{\tau}^{\prime \prime}-\mathcal{E}^{\prime} a_{\tau}^{\prime} \hat{e}_{\tau}^{\prime}\right)\right]=0,
$$

donde se han usado las expresiones para $\vec{E}_{I}, \vec{E}_{I I}$ que derivan de las ecs. (30), (31), (32) y (43).

Las condiciones que vienen de las ecuaciones de Maxwell restantes se obtienen de forma similar. En general, por medio de las ecuaciones anteriores y la definición 
presentada anteriormente de los vectores de polarización correspondientes, se asegura que las soluciones propuestas satisfacen las ecuaciones en cada región separadamente. Así, cuando la solución es sustituida en las ecuaciones de Maxwell, solamente los términos que surjan de las derivadas actuando en la función $\Theta$, las cuales son proporcionales a la función $\delta$, no son automáticamente cero. Demandar que ellos sean cero también produce las relaciones requeridas. Procediendo de esta manera se encuentra que la ec. (4) produce de nuevo la ec. (45) y las dos condiciones que quedan que complementan las ecs. (46) y (49) son

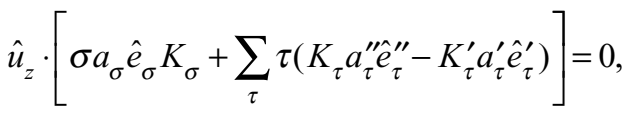

$$
\begin{aligned}
& \hat{u}_{z} \times\left[\sigma a_{\sigma} \varepsilon v_{\sigma} \hat{e}_{\sigma}+\sum_{\tau} \tau\left(a_{\tau}^{\prime \prime} \varepsilon v_{\tau} \hat{e}_{\tau}^{\prime \prime}-a_{\tau}^{\prime} \varepsilon^{\prime} v_{\tau}^{\prime} \hat{e}_{\tau}^{\prime}\right)\right]=0
\end{aligned}
$$

Al escribir estas condiciones, se ha usado la relación

$$
i \hat{k}_{\sigma} \times \hat{e}_{\sigma}=\sigma \hat{e}_{\sigma}
$$

Además se ha considerado el hecho que, para cualquier onda (incidente, refractada y reflejada), el producto de sus números de ondas por sus velocidades es igual a $\omega$, la que es la misma para todas ellas.

\section{Las condiciones independientes}

La tarea es usar las condiciones dadas en las ecs. (46), (49), (50) y (51) para obtener, para una amplitud incidente dada $a_{\sigma}$, las amplitudes reflejada y transmitida $a_{\tau}^{\prime}, a_{\tau}^{\prime \prime}$. Existen así un total de cuatro variables desconocidas por ser resueltas. Por otro lado, a primera vista, el número de condiciones parecen ser seis. Mientras las ecs. (49) y (50) son ecuaciones escalares, las relaciones en las ecs. (51) y (52) son vectoriales. Siendo un problema de dos dimensiones, como se ha visto, cada una de las relaciones de vector produce dos condiciones, para un total de seis. Por lo tanto, la solución para $a_{\tau}^{\prime}, a_{\tau}^{\prime \prime}$ es algebraicamente sobredeterminada y existe una solución no trivial solamente si son satisfechas condiciones auxiliares adicionales. Como se verá, éstas son sólo las relaciones de tipo Snell dadas en las ecs. (46) y (49) y las relaciones de dispersión de la ec. (18). Puesto de otra manera, cuando la ec. (18) así como las ecs. (46) y (49) son satisfechas, dos de las seis relaciones implicadas por el conjunto de condiciones de frontera son redundantes, dejando sólo cuatro ecuaciones independientes para las cuatro desconocidas $a_{\tau}^{\prime}, a_{\tau}^{\prime \prime}$.
En el caso correspondiente a un medio ordinario, no quiral, para un valor dado de la componente transversal del vector de onda y una frecuencia dada, la componente longitudinal del vector de onda en cada una de las ondas reflejada y refractada tiene un valor único. Así, la solución escrita en la ec. (31) en efecto colapsa a la forma dada en la ec. (30). No obstante, el problema es similar al declarado anteriormente. A saber, para una amplitud incidente dada $\vec{a}$, se desea conocer que son los coeficientes $\vec{b} \mathrm{y}$ $\vec{c}$. Siendo un problema de dos dimensiones como ya se discutió, entonces para un $\vec{a}$ arbitrario (pero definido) en el plano $\hat{e}_{1,2}$, existen cuatro desconocidos, representados por los dos componentes de $\vec{b}$ en el plano $\hat{e}_{1,2}^{\prime \prime}$ y los dos componentes de $\vec{c}$ en el plano $\hat{e}_{1,2}^{\prime}$. Aún más, siendo el problema lineal (las condiciones de frontera son ecuaciones lineales en las amplitudes), se puede avanzar para encontrar las soluciones particulares correspondientes al caso $\vec{a}=\hat{e}_{1} \mathrm{y}$, separadamente, $\vec{a}=\hat{e}_{2}$, y la solución general para una elección arbitraria de $\vec{a}$ se obtiene por superposición lineal de esas soluciones particulares. Este es de hecho el procedimiento seguido en el libro de Jackson [1]. La razón fundamental de por qué una solución puede ser obtenida del todo, es el hecho de que existen cuatro relaciones algebraicas independientes (que vienen de las condiciones de frontera) entre las cuatro desconocidas que hemos identificado.

Regresando al problema de este trabajo, esos mismos principios generales se mantienen. A saber, mientras algunas características y detalles de las soluciones no son aplicables o relevantes, la propiedad de linealidad, principio de superposición y el hecho de que el problema está algebraicamente bien definido, también se aplican. La diferencia fundamental, en lo que a los detalles y manipulaciones algebraicas concierne, es que en el presente caso no es útil construir la solución general por la superposición de las soluciones particulares obtenidas para los casos $\vec{a}=\hat{e}_{1,2}$ y descomponer las ondas reflejada y refractada en términos de las componentes de polarización lineal. En este caso, la polarización circular base es, como se ha demostrado, la apropiada de usar.

Para el álgebra del vector restante se usaran las siguientes relaciones

$$
\begin{aligned}
& \hat{u}_{z} \cdot \hat{e}_{\sigma}=-\frac{1}{\sqrt{2}} i \sigma \operatorname{sen} \alpha, \\
& \hat{u}_{z} \cdot \hat{e}_{\tau}^{\prime}=-\frac{1}{\sqrt{2}} i \tau \operatorname{sen} \alpha_{\tau}^{\prime}, \\
& \hat{u}_{z} \cdot \hat{e}_{\tau}^{\prime \prime}=-\frac{1}{\sqrt{2}} i \tau \operatorname{sen} \alpha_{\tau}^{\prime \prime},
\end{aligned}
$$


$\mathrm{y}$

$$
\begin{aligned}
& \hat{u}_{z} \times \hat{e}_{\sigma}=\frac{1}{\sqrt{2}}\left(\hat{u}_{y}-i \sigma \cos \alpha \hat{u}_{x}\right), \\
& \hat{u}_{z} \times \hat{e}_{\tau}^{\prime}=\frac{1}{\sqrt{2}}\left(\hat{u}_{y}-i \tau \cos \alpha_{\tau}^{\prime} \hat{u}_{x}\right), \\
& \hat{u}_{z} \times \hat{e}_{\tau}^{\prime \prime}=\frac{1}{\sqrt{2}}\left(\hat{u}_{y}+i \tau \cos \alpha_{\tau}^{\prime \prime} \hat{u}_{x}\right) .
\end{aligned}
$$

Usando estas reglas de multiplicación, las ecuaciones anteriores., implican, en ese orden, las siguientes seis relaciones

$$
\begin{aligned}
& \sum_{\tau}\left(a_{\tau}^{\prime}-a_{\tau}^{\prime \prime}\right)=a_{\sigma}, \\
& \sum_{\tau} \tau\left(a_{\tau}^{\prime} \cos \alpha_{\tau}^{\prime}+a_{\tau}^{\prime \prime} \cos \alpha_{\tau}^{\prime \prime}\right)=\sigma a_{\sigma} \cos \alpha, \\
& \sum_{\tau}\left(K_{\tau}^{\prime} a_{\tau}^{\prime} \operatorname{sen} \alpha_{\tau}^{\prime}-K_{\tau} a_{\tau}^{\prime \prime} \operatorname{sen} \alpha_{\tau}^{\prime \prime}\right)=a_{\sigma} K_{\sigma} \operatorname{sen} \alpha, \\
& \sum_{\tau} \tau\left(\varepsilon^{\prime} a_{\tau}^{\prime} \operatorname{sen} \alpha_{\tau}^{\prime}-\varepsilon a_{\tau}^{\prime \prime} \operatorname{sen} \alpha_{\tau}^{\prime \prime}\right)=\sigma a_{\sigma} \varepsilon \operatorname{sen} \alpha, \\
& \sum_{\tau}^{\tau} \tau\left(a_{\tau}^{\prime} \varepsilon^{\prime} v_{\tau}^{\prime}-a_{\tau}^{\prime \prime} \varepsilon v_{\tau}\right)=\sigma a_{\sigma} \varepsilon v_{\sigma}, \\
& \sum_{\tau}\left(a_{\tau}^{\prime} \varepsilon^{\prime} v_{\tau}^{\prime} \cos \alpha_{\tau}^{\prime}+a_{\tau}^{\prime \prime} \varepsilon v_{\tau} \cos \alpha_{\tau}^{\prime \prime}\right)=a_{\sigma} \varepsilon v_{\sigma} \cos \alpha .
\end{aligned}
$$

Si ahora se usa la ley de Snell [ecs. (39) y (40)], es fácil ver que la tercera ecuación se vuelve idéntica a la primera, y la cuarta idéntica a la quinta. Así, se ha quedado con cuatro ecuaciones independientes las que, para una fácil referencia en lo que sigue, se presentan más abajo:

$$
\begin{aligned}
& \sum_{\tau}\left(a_{\tau}^{\prime}-a_{\tau}^{\prime \prime}\right)=a_{\sigma} \\
& * \sum_{\tau} \tau\left(a_{\tau}^{\prime} \cos \alpha_{\tau}^{\prime}+a_{\tau}^{\prime \prime} \cos \alpha_{\tau}^{\prime \prime}\right)=\sigma a_{\sigma} \cos \alpha \\
& * \sum_{\tau} \tau\left(a_{\tau}^{\prime} \varepsilon^{\prime} v_{\tau}^{\prime}-a_{\tau}^{\prime \prime} \varepsilon v_{\tau}\right)=\sigma a_{\sigma} \varepsilon v_{\sigma} \\
& \sum_{\tau}\left(a_{\tau}^{\prime} \varepsilon^{\prime} v_{\tau}^{\prime} \cos \alpha_{\tau}^{\prime}+a_{\tau}^{\prime \prime} \varepsilon v_{\tau} \cos \alpha_{\tau}^{\prime \prime}\right)=a_{\sigma} \varepsilon v_{\sigma} \cos \alpha
\end{aligned}
$$

Estas ecuaciones deben ser resueltas para los cuatro variables desconocidas $a_{\tau}^{\prime}$ y $a_{\tau}^{\prime \prime}(\operatorname{con} \tau= \pm)$, para cualquier amplitud incidente $a_{\sigma}$ dada.

\section{Solución general}

Otros casos especiales pueden ser tratados en forma similar, tal como en el caso de la incidencia desde un medio quiral a uno ordinario, incluyendo la situación particular de incidencia normal. Sin embargo, no se continuará más por esa línea, y se dará directamente la solución a la ec. (56) para las amplitudes reflejada y refractada, en el caso general. Para este propósito, usamos la notación abreviada $c_{0} \equiv \cos \alpha, c_{ \pm}^{\prime} \equiv \cos \alpha_{ \pm}^{\prime}$ y $c_{ \pm}^{\prime \prime} \equiv \cos \alpha_{ \pm}^{\prime \prime}$ para el coseno de varios ángulos. Entonces, definiendo

$$
\begin{aligned}
D= & \left(c_{+}^{\prime} c_{-}^{\prime}+c_{+}^{\prime \prime} c_{-}^{\prime \prime}\right)\left(\varepsilon^{\prime} v_{+}^{\prime}+\varepsilon^{\prime} v_{-}^{\prime}\right)\left(\varepsilon v_{+}+\varepsilon v_{-}\right)+ \\
& +\left(c_{+}^{\prime} c_{-}^{\prime \prime}+c_{-}^{\prime} c_{+}^{\prime \prime}\right)\left(\varepsilon^{\prime} v_{-}^{\prime}+\varepsilon v_{+}\right)\left(\varepsilon^{\prime} v_{+}^{\prime}+\varepsilon v_{-}\right)+ \\
& +\left(c_{+}^{\prime} c_{+}^{\prime \prime}+c_{-}^{\prime} c_{-}^{\prime \prime}\right)\left(\varepsilon^{\prime} v_{+}^{\prime}-\varepsilon v_{+}\right)\left(\varepsilon^{\prime} v_{-}^{\prime}-\varepsilon v_{-}\right)
\end{aligned}
$$

la solución está dada por

$$
\begin{aligned}
\frac{a_{\tau}^{\prime}}{a_{\sigma}} D= & \left(\sigma \varepsilon v_{\sigma}+\tau \varepsilon^{\prime} v_{-\tau}^{\prime}\right)\left(\varepsilon v_{+}+\varepsilon v_{-}\right)\left(\sigma c_{0} c_{-\tau}^{\prime}+\tau c_{+}^{\prime \prime} c_{-}^{\prime \prime}\right)+ \\
& +\left(\sigma \varepsilon v_{\sigma}+\varepsilon v_{-}\right)\left(\varepsilon^{\prime} v_{-\tau}^{\prime}+\tau \varepsilon v_{+}\right)\left(\sigma c_{0} c_{-}^{\prime \prime}+\tau c_{-\tau}^{\prime} c_{+}^{\prime \prime}\right)+ \\
& +\left(\sigma \varepsilon v_{\sigma}-\varepsilon v_{+}\right)\left(\varepsilon^{\prime} v_{-\tau}^{\prime}-\tau \varepsilon v_{-}\right)\left(\sigma c_{0} c_{+}^{\prime \prime}+\tau c_{-\tau}^{\prime} c_{-}^{\prime \prime}\right)
\end{aligned}
$$

$$
\begin{aligned}
\frac{a_{\tau}^{\prime \prime}}{a_{\sigma}} D= & \left(\sigma \varepsilon v_{\sigma}+\tau \varepsilon v_{-\tau}\right)\left(\varepsilon^{\prime} v_{+}^{\prime}+\varepsilon^{\prime} v_{-}^{\prime}\right)\left(\sigma c_{0} c_{-\tau}^{\prime \prime}-\tau c_{+}^{\prime} c_{-}^{\prime}\right)+ \\
& +\left(\sigma \varepsilon v_{\sigma}+\tau \varepsilon^{\prime} v_{-\tau}^{\prime}\right)\left(\varepsilon^{\prime} v_{\tau}^{\prime}+\varepsilon v_{-\tau}\right)\left(\sigma c_{0} c_{-\tau}^{\prime}-\tau c_{\tau}^{\prime} c_{-\tau}^{\prime \prime}\right)- \\
& -\left(\sigma \varepsilon v_{\sigma}-\tau \varepsilon^{\prime} v_{\tau}^{\prime}\right)\left(\varepsilon^{\prime} v_{-\tau}^{\prime}-\varepsilon v_{-\tau}\right)\left(\sigma c_{0} c_{\tau}^{\prime}-\tau c_{-\tau}^{\prime} c_{-\tau}^{\prime \prime}\right)
\end{aligned}
$$

Estas fórmulas pueden ser escritas en varias formas alternativas, en términos del ángulo de incidencia y los índices refractivos, usando la ley de Snell. En los límites apropiados, las ecuaciones se reducen a las fórmulas de casos especiales encontradas en artículos de otros autores [9-14].

\section{CONCLUSIONES}

Las propiedades electromagnéticas de un medio que exhibe quiralidad (también llamado actividad óptica), pero el cual es en otros aspectos lineal, homogéneo e isotrópico, puede ser descrito en términos de los dos parámetros usuales $\varepsilon$ y $\mu$ que representan las funciones dieléctrica y permeabilidad, y un parámetro adicional $T$ o $\zeta$ que es indicativo de la propiedad de quiralidad. Como hemos discutido aquí y en las referencias citadas, tal parametrización en términos de sólo una función adicional, es completa y mínima. En el caso de un medio infinito (sin límites), una onda electromagnética exhibe el fenómeno conocido como "actividad óptica natural", la cual se debe al hecho de que los dos estados polarizados circularmente viajan con diferente velocidad si $T$ o $\zeta$ es distinto de cero. 
En este trabajo se ha considerado en detalle varios aspectos de la propagación de la onda, en un medio que está hecho de dos medios semiinfinitos, uno o ambos pueden ser quirales, los cuales están separados por un plano interfaz, imitando el procedimiento que se aplica al problema análogo que envuelve dieléctricos ordinarios, la aproximación presentada está basada en escribir la solución de onda plana que satisfaga las ecuaciones de Maxwell en cada región, suplementado por las condiciones apropiadas de frontera en la interfaz.

Se consideró primero varios aspectos cinéticos de la solución los que son independientes de la naturaleza detallada de las condiciones de frontera. En particular, se obtuvo la ley de Snell y la condición para el efecto de reflexión interna total, en la forma que aplica a la presente situación. Los resultados generales obtenidos en este trabajo sirven como base para resolver casos particulares como: medios no quirales, incidencia de medio normal a quiral, incidencia normal entre dos medios quirales, etc.; y para abordar problemas de quiralidad en sistemas complejos tales como son los metamateriales quirales, la electrodinámica fractal y la difracción quiral (trabajo en proceso de elaboración). Estos trabajos podrían encontrar aplicación en diseños de microondas en muy alta frecuencia, guías de ondas fraccionales y líneas de transmisión no simétricas.

\section{AGRADECIMIENTOS}

Uno de los autores (HTS) agradece el financiamiento parcial para este trabajo por parte del Proyecto Mayor No 8721-06 de la Universidad de Tarapacá, Arica-Chile.

\section{REFERENCIAS}

[1] J.D. Jackson. “Classical Electrodynamics”. Wiley. New York. 1975.

[2] S. Ichimaru. "Statistical Plasma Physics I". Addison-Wesley. New York. 1992.

[3] T. Verbiest, M. Kauranen and André Persoons. "Light-Polarization-Induced Optical Activity". Phys. Rev. Lett. Vol. 82, pp. 3601-3604. 1999.

[4] E. Charney. "The Molecular Basis of Optical Activity”. Wiley. New York. 1979.
[5] D.R. Smith, W.J. Padilla, D.C. Vier, S.C. NematNasser and S. Schultz. "Composite Medium with Simultaneously Negative Permeability and Permittivity". Phys. Rev. Lett. Vol. 84, pp. 41844187. 2000.

[6] E.U. Condon. "Theories of optical rotatory power". Rev. Mod. Phys. Vol. 9. 1937.

[7] P. Drude. "The Theory of Optics". Dover Publications, INC. New York. 1959.

[8] E.J. Post. "Formal Structure of Electromagnetics: General Covariance and Electromagnetics". NorthHolland. Amsterdam. 1962.

[9] A. Lakhtakia, V.V. Varadan, V.K. Varadan. "A parametric study of microwave reflection characteristics of a planar achiral-chiral interface". IEEE Trans. Electromag. Compat. Vol. EMC-28 No 2, pp. 90-95. 1986.

[10] J. F. Nieves and P. B. Pal. "The Third Electromagnetic Constant of an Isotropic Medium”. Am. J. Phys. Vol. 62, pp. 207. 1994.

[11] J.A. Kong. "Theorems of bianisotropic media".

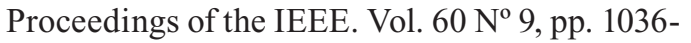
1046. Septiembre 1972.

[12] C. Krowne. "Electromagnetic theorems for complex anisotropic media". IEEE Transactions on Antennas and Propagation. Vol. $32 \mathrm{~N}^{\mathrm{o}} 11$, pp. 1224-1230. Noviembre 1984.

[13] J.C. Monzon. "Radiation and scattering in homogeneous general biisotropic regions". IEEE Trans. Antennas Propagat. Vol. 38, pp. 227-235. 1990.

[14] P. Hillion. "How do focus wave modes propagate across a discontinuity in a medium?". Optik. Vol. $93 \mathrm{~N}^{\mathrm{o}}$ 2, pp. 67-72. 1993.

[15] H. Torres Silva, P.H. Sakanaka, N. Reggiani and C. Villarroel. "Electromagnetic properties of a chiral-plasma-media". Pramana Journal of Physics. Vol. 48, pp. 1-12. 1997. 